\title{
Efeitos das distâncias e recursos da empresa no desempenho exportador de empresas
}

\author{
Givanildo Silva ${ }^{1 A}$, Mohamed Amal ${ }^{A}$, Gérson Tontini ${ }^{A}$ e Gustavo da Rosa Borges ${ }^{B}$ \\ ${ }^{A}$ Fundação Universidade Regional de Blumenau - FURB, Blumenau, SC, Brasil \\ ${ }^{B}$ Universidade Federal do Pampa - UFP, Dom Pedrito, RS, Brasil
}

\begin{tabular}{l} 
DETALHES DO ARTIGO \\
Histórico do artigo: \\
Recebido em 14 de janeiro de 2015 \\
Aceito em 27 de julho de 2015 \\
Disponível online em 19 de dezembro de 2015 \\
Sistema de Revisão “Double Blind Review” \\
Editor científico: \\
Felipe Mendes Borini \\
\hline
\end{tabular}

\section{Palavras-chaves:}

Distância institucional

Determinantes de exportação

Recursos da empresa

\begin{abstract}
RESUMO
O objetivo deste artigo é avaliar o efeito da distância sobre o desempenho exportador das empresas de Santa Catarina e em que medida tal efeito é moderado pelas características dos recursos organizacionais. Foi utilizada regressão linear múltipla e análise de variância a partir de levantamento da percepção dos gestores de exportação em uma amostra final de 49 empresas produtoras exportadoras. Os constructos exibiram validade interna e possibilitam a análise dos dados. Os resultados apresentaram apenas evidências sobre o efeito da distância psíquica, apontando para uma relação positiva com o desempenho de exportação. Além disso, a estimação do modelo mostrou que os recursos da organização moderam a relação entre distância e desempenho exportador. Finalmente, o estudo mostra também que a equipe de exportação e a estrutura organizacional moderam o efeito da distância sobre o desempenho e expectativa futura de desempenho das empresas
\end{abstract}

(C) 2015 Internext | ESPM. Todos os direitos reservados!

\section{Introdução}

Distância cultural é relacionada às semelhanças nos valores, normas e religião que facilitam a interação e a comunicação (HUTZSCHENREUTER; VOLL; VERBEKE, 2011). As multinacionais precisam acessar e utilizar o conhecimento relacionado aos costumes locais e outras condições externas afetadas pela cultura local (BARKEMA; BELL; PENNINGS, 1996). Uma maior distância cultural aumenta os desafios e o tempo necessário para a empresa se ajustar de forma eficaz no novo ambiente, ou seja, pode-se incorrer em maiores custos de ajuste dinâmico (HUTZSCHENREUTER; VOLL; VERBEKE, 2011).

Além de considerar as diferenças culturais, a distância institucional trata de restrições concebidas pelas pessoas por meio de estruturas políticas, interações econômicas e sociais, e pode ser classificada em forças informais: valores, crenças, sanções, tabus, costumes, tradições, códigos de conduta, comportamentos comuns e práticas da sociedade; e regras formais: constituições, leis, regras, sanções e direitos de propriedade (NORTH, 1991). A distância institucional é influenciada pelas percepções dos gestores, por seus entendimentos e opiniões sobre a política e as condições institucionais dos países estrangeiros (GHEMAWAT, 2001).

Em estudo realizado na China, o desempenho superior das exportações está alinhado com a visão baseada em recursos e a distância institucional (HE; BROUTHERS; FILATOTCHEV, 2012). No Brasil, verificou-se efeito negativo moderador do efeito da distância psíquica na rentabilidade das exportações, que pode ser explicada considerando que alguns custos tendem a ser menores nos países desenvolvidos, enquanto outros tendem a serem mais elevados (CARNEIRO; ROCHA; SILVA, 2011).

$\mathrm{Na}$ visão baseada em recursos (VBR), as capacidades podem ser vistas como conjuntos de ativos tangíveis e intangíveis, incluindo as competências de gestão da empresa, processos, 
rotinas organizacionais, informações e conhecimentos. Nos negócios internacionais, a VBR ajuda a especificar a natureza dos recursos necessários para superar o passivo da internacionalização (liability of foreignness- LOF) e indica um método para investigar os recursos referentes à definição de produtos e diversificação internacional (BARNEY; WRIGHT; KETCHEN, 2001).

O presente trabalho tem como objetivo de analisar os determinantes de desempenho exportador a partir dos efeitos das distâncias psíquica, cultural e institucional, e pela perspectiva da visão baseada em recursos. Para atender este objetivo, a pergunta de pesquisa deste estudo é: Quais os efeitos das distâncias sobre o desempenho exportador de empresas? E, em que medida, tais efeitos são moderados pelos recursos da empresa?

Este artigo compõe-se de cinco seções. Após esta introdução apresenta-se a revisão de literatura e as hipóteses de pesquisa. A terceira seção descreve o método, técnica de pesquisa, constructos, variáveis, modelo moderador e amostra. Na quarta seção são expostos e discutidos os resultados das análises de regressão linear múltipla e rotação Varimax. As considerações finais, os impactos perante demais estudos e limitações desta investigação são mostrados ao final.

\section{Referencial Teórico 2.1 Desempenho exportador}

Os conceitos apresentados por Platchek, Floriani e Borini (2012, p. 73) explicam o desempenho exportador, pois citam Aulakh, Kotabe e Teegen (2000), que avaliaram três dimensões, sendo, "estratégia competitiva, nível de padronização versus adaptação da oferta e grau de diversificação geográfica das exportações". Os autores também mencionaram Leonidou et al. (2002), que decompuseram em três níveis de análise, sendo, "influência das variáveis de estratégia de exportação sobre o desempenho em geral, efeito das variáveis de estratégia de exportação sobre medidas específicas de desempenho e variações dos efeitos das variáveis de estratégia de exportação sobre o desempenho"; bem como apontaram Madsen (1989), que selecionou fatores que explanassem o desempenho exportador, sendo, "a estratégia de marketing de exportação e as características da empresa".

O desempenho exportador pode ser mensurado pelo volume de receitas de exportação, lucratividade das exportações, crescimento das receitas da empresa, intensidade de exportação, experiência internacional da empresa, grau de diversificação geográfica, estratégia de marketing de exportação, atratividade do mercado externo, barreiras à exportação, distância geográfica, distância psíquica, expectativas e características dos gerentes, entre outros (PLATCHEK; FLORIANI; BORINI, 2012).

Pesquisa com 195 empresas industriais exportadoras da China concluiu que o desempenho superior das exportações está alinhado com fatores baseados em recursos e a distância institucional. A análise empírica sugere que, depois de considerar a influência moderadora da distância institucional, alinhando recursos internos com o canal de exportação, há maior desempenho das exportações. Os resultados sugerem que a distância institucional entre os mercados domésticos e de exportação modera esta relação, aumentando valor ao se usar canais hierárquicos quando as empresas possuem fortes capacidades de orientação para o mercado ou a necessidade de parceiros quando as empresas não têm tais capacidades (HE; BROUTHERS; FILATOTCHEV, 2012).

Estudo realizado com amostra final de 389 grandes exportadores brasileiros de produtos manufaturados verificou efeito negativo moderado da distância psíquica na rentabilidade das exportações, que pode ser explicado considerando que alguns custos tendem a ser menores nos países desenvolvidos (transporte e questões legais), enquanto outros tendem a serem mais elevados (publicidade e promoção). Também era esperada uma relação negativa dos efeitos da distância, contudo não foram encontrados efeitos significativos. Uma possível explicação é que os gestores possam prestar mais atenção às operações em mercados psiquicamente distantes, com o objetivo de se preparar para dificuldades maiores do que o encontrado (CARNEIRO; ROCHA; SILVA, 2011).

\subsection{Distância psíquica e o modelo de Uppsala revisitado}

A distância psíquica foi observada na tendência de países concentrarem suas relações comerciais em países mais próximos, em que os contatos estrangeiros já foram pessoalmente estabelecidos e desenvolvidos, muito além do que a distância econômica pode explicar. Desta forma, a distância psíquica foi conceituada pelo seu aspecto subjetivo em relação a distância econômica objetiva, que trata 
dos custos de movimentação de bens físicos (transporte, tarifas e outros) (BECKERMAN, 1956).

A distância psíquica trata da percepção implícita de como o indivíduo avalia as diferenças entre os países estrangeiros por meio das formas de relações familiares, hábitos e costumes (DICHTL; KOEGLMAYR; MUELLER, 1990). Extrapolando para a teoria de negócios internacionais, a distância psíquica trata da percepção média referente a determinado país, que contribui para interpretar o comportamento médio das empresas daquele país, sendo que as normas de interação e comunicação empresarial podem ser altamente influenciadas pelo nível de desenvolvimento econômico dos países (DOW; KARUNARATNA, 2006).

O processo de internacionalização de empresas de Uppsala propõe que as empresas multinacionais iniciam suas atividades exportadoras pelos países mais próximos antes de buscarem mercados em regiões com maior distância psíquica (JOHANSON; VAHLNE, 1977). Este modelo foi revisado considerando que não importam as adversidades de ser uma empresa estrangeira em um país distante, mas sim o apoio recebido de um parceiro local. Pode ser difícil de iniciar a expansão internacional caso a multinacional não tenha aderência as redes de negócios em contextos locais desconhecidos. Desta forma, as relações existentes em um novo ambiente são mais importantes do que as distâncias entre os países da matriz e da filial (JOHANSON; VAHLNE, 2009).

Portanto, quanto mais familiar for o país estrangeiro, menor será a distância psíquica. Contudo, ao se subestimar o grau de adaptação necessária ao entrar em um mercado que se percebe como familiar e semelhante ao mercado doméstico, pode-se incorrer no paradoxo distância psíquica decorrente de assimetrias nas percepções psíquicas de distância. Por exemplo, a expansão de empresas dos EUA sobre o Canadá, bem como, no resto do mundo, relacionado a grandes países e seus pequenos vizinhos (HÅKANSON; AMBOS, 2010).

Acredita-se que as distâncias psíquicas, como convencionalmente medidas pelas distâncias culturais, não são simétricas, ou seja, as distâncias percebidas de $A$ para $B$ e de $B$ para $A$ não são necessariamente iguais (SHENKAR, 2001). Dados de pesquisa descritiva confirmam a existência de considerável assimetria e a análise estatística sugere algumas das fontes desse problema (HÅKANSON; AMBOS, 2010).
O fenômeno da empresa "nascida global" que rapidamente entra em mercados muito distantes sugere, por exemplo, que a variância nas percepções de distâncias psíquicas de gestores individuais talvez aumentasse, dramaticamente, ao longo do tempo (HÅKANSON; AMBOS, 2010). Outra abordagem teórica e operacionalmente mais simples é medir a distância psíquica direta e, posteriormente, avaliar em que medida a distância cultural, as diferenças linguísticas e outros fatores, impactam nestas percepções (SOUSA; BRADLEY, 2006).

\subsection{Distância cultural}

Uma das principais dificuldades relacionadas a expansão internacional é a distância entre os novos contextos locais e os ambientes em que a organização já atua, sendo que a dimensão crítica é a distância cultural (GHEMAWAT, 2001). A distância cultural é relacionada às semelhanças nos valores, normas e religião que facilitam a interação e a comunicação entre pessoas de países distintos, por meio de significados compartilhados, entendimentos tácitos, expectativas de contextos e de comportamentos. Desta forma, na medida em que as culturas são diferentes, tais entendimentos ficam debilitados e a comunicação torna-se mais difícil (HUTZSCHENREUTER; VOLL; VERBEKE, 2011). Quanto maior a distância cultural entre os países exportadores e importadores, mais difícil será identificar e interpretar corretamente as informações disponíveis sobre o mercado externo (SOUSA; BRADLEY, 2006). As multinacionais precisam acessar e utilizar os conhecimentos relacionados aos costumes locais e outras condições externas afetadas pela cultura local (BARKEMA; BELL; PENNINGS, 1996).

Pesquisas indicam que as percepções, comportamentos e crenças das pessoas são orientados pela sua cultura em nível nacional, ou seja, os indivíduos de países diferentes pensam e agem de forma diversa, devido a sua cultura (KIRKMAN; LOWE; GIBSON, 2006; LEUNG et al., 2005). Portanto, negócios internacionais em culturas distantes precisam considerar a gestão de conflitos, tomada de decisões, interação dos membros das equipes, fornecedores e clientes, que apresentam diferentes crenças, percepções e processos cognitivos (HUTZSCHENREUTER; VOLL; VERBEKE, 2011).

A diversidade cultural existente, caracterizada pelo conjunto de contextos locais em que a organização opera atualmente, tem efeito negativo sobre a taxa de expansão internacional, pois aumenta 
a complexidade da governança interna e ambiental, no tocante a gerenciar as relações internas da rede de empresas da multinacional, bem como as relações com as partes interessadas externas e, pode, portanto, desestimular o aumento ainda maior da distância cultural (HUTZSCHENREUTER; VOLL; VERBEKE, 2011).

\subsection{Distância institucional}

A premissa básica da teoria institucional é que as tendências das empresas em relação a conformidade com normas e tradições predominantes em cada contexto social leva a homogeneidade entre as empresas, em suas estruturas e atividades, e que as bem sucedidas são aquelas que ganham apoio e legitimidade, em conformidade com as pressões Sociais (MEYER; ROWAN, 1977; OLIVER, 1997).

Instituições são restrições concebidas pelas pessoas, por meio de estruturas políticas e interações econômicas e sociais, podendo ser classificadas em forças informais (valores, crenças, sanções, tabus, costumes, tradições, códigos de conduta, comportamentos comuns e práticas da sociedade) e regras formais (constituições, leis, regras, sanções, direitos de propriedade), com o objetivo de criar ordem e reduzir a incerteza econômica (NORTH, 1991).

Outra perspectiva de análise das instituições pode ser a área de política (corrupção, transparência), a legislação (liberalização econômica, regime regulatório) e a sociedade (normas éticas, atitudes em relação ao empreendedorismo). As instituições podem ser analisadas como variáveis independentes, em uma visão baseada em estratégia institucional, que se centra na interação dinâmica entre instituições e organizações, e considera-se as escolhas estratégicas como resultado dessa interação (PENG; WANG; JIANG, 2008). As instituições nacionais fornecem uma estrutura de incentivos para a economia que, por meio da evolução, moldam a direção da mudança para o crescimento econômico, estagnação ou declínio (NORTH, 1991).

A distância institucional é influenciada pelas percepções dos gestores, por seus entendimentos e opiniões sobre a política, e as condições institucionais dos países estrangeiros. Essas diferenças relacionamse ao ambiente regulatório de um país, que pode ser transparente e com estruturas de governança eficientes. Desta forma, mais fáceis de compreender e se relacionar. Ou, por outro lado, com instituições políticas e judiciais pouco desenvolvidas, onde os costumes podem ser regidos por regras informais e convenções que podem parecer estranhas, ineficientes ou mesmo corruptas e imorais (GHEMAWAT, 2001).

As atividades das multinacionais podem sofrer pressões institucionais em nível interorganizacional, que surgem de fontes externas, tais como governos, mercados e sociedade (HOFFMAN, 2001), ou em nível organizacional, a partir da cultura, sistemas de crenças compartilhadas e processos políticos (DIMAGGIO; POWELL, 1983), e também dos acionistas (HENRIQUES; SADORSKY, 1996).

Todos os atores institucionais podem impor diferentes pressões coercivas, miméticas e normativas sobre os gestores. As pressões coercitivas têm forças impostas pelos governos. As pressões miméticas ocorrem por meio da imitação organizacional ou por modelagem de normas e práticas no campo institucional da organização. E, as pressões normativas têm suas origens na profissionalização da indústria ou de membros do setor que tentam definir as condições e métodos para seu trabalho, buscando legitimar sua autonomia profissional (DIMAGGIO; POWELL, 1983; OLIVER, 1997).

A partir dos estudos sobre distância e desempenho exportador, apresentamos a primeira hipótese desta investigação que, logo em seguida, é dividida nas distâncias psíquica, cultural e institucional:

- Hipótese 1: Quanto maior a distância entre o país de origem da empresa exportadora e o país de destino, menor é o desempenho exportador da organização.

- Hipótese 1a: Quanto maior a distância psíquica entre o país de origem da empresa exportadora e o país de destino, menor é o desempenho exportador da organização.

- Hipótese 1b: Quanto maior a distância cultural entre o país de origem da empresa exportadora e o país de destino, menor é o desempenho exportador da organização.

- Hipótese 1c: Quanto maior a distância institucional entre o país de origem da empresa exportadora e o país de destino, menor é o desempenho exportador da organização. 


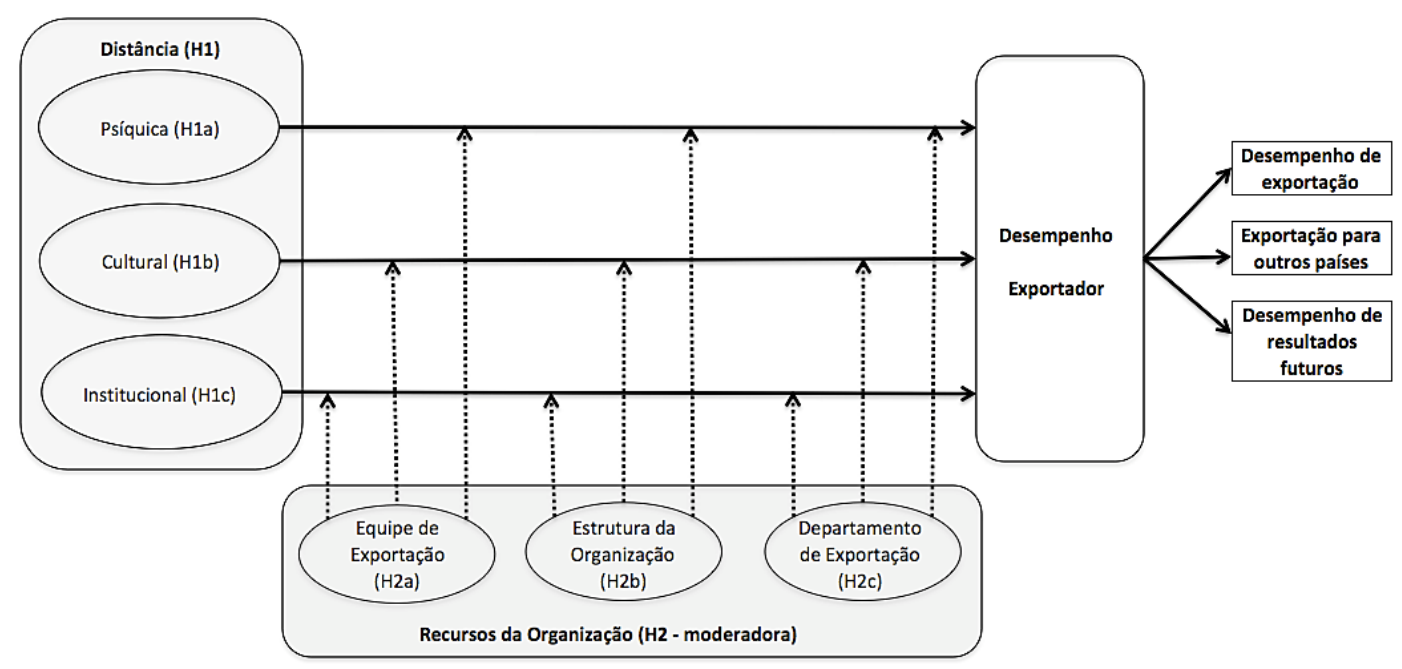

Figura 1. Descrição gráfica do modelo Fonte: Autores

\subsection{Visão Baseada em Recursos}

Os estudos sobre gestão estratégica sugerem que as empresas obtêm vantagens competitivas sustentáveis por meio da implementação de estratégias que exploram suas forças e evitam suas fraquezas internas, enquanto neutralizam as ameaças externas. Os quatro indicadores empíricos dos recursos potenciais das organizações para gerar vantagem competitiva sustentável proposta são: ser valioso, raro entre os concorrentes, difícil de imitar e sem substitutos estrategicamente equivalentes. O papel dos gestores na interpretação e análise das capacidades dos recursos controlados pela organização é fundamental para a obtenção de vantagem competitiva sustentada (BARNEY, 1991).

A visão baseada em recursos não considera o contexto social em que as decisões são tomadas, referente a seleção e incorporação de recursos com objetivo de heterogeneidade da organização. Desta forma, faz-se necessário um modelo de vantagem competitiva sustentável que considere a convergência entre a teoria institucional e a visão baseada em recursos. Em nível individual, o modelo proposto por Oliver (1997) analisa a racionalidade normativa, referente a custos cognitivos e conformidade com as tradições. Em nível organizacional, consideram-se os fatores institucionais, tais como, cultura e política adotadas internamente. E, em nível social, tratam-se das pressões isomórficas, no tocante ao governo, profissões, alianças entre as empresas, entre outros (OLIVER, 1997).
A visão baseada em recursos contribui para especificar a natureza dos recursos necessários para superar a responsabilidade do estrangeirismo (BARNEY; WRIGHT; KETCHEN, 2001). A multinacional deve recombinar sua base de recursos existentes aqueles necessários no ambiente exterior, o que é um processo demorado dado a responsabilidade do parceiro local (JOHANSON; VAHLNE, 2009). O desafio é, portanto, desenvolver experiência gerencial que possa suportar tal recombinação de recursos e a coordenação em vários contextos. A empresa multinacional também deve aumentar sua base de recursos existentes e construir novas capacidades organizacionais, tanto para gerenciar a complexa estrutura interna, bem como a sua rede externa (MEYER; MUDAMBI; NARULA, 2011).

Conclui-se que os gestores precisam examinar cuidadosamente antes de empreender muitos projetos de expansão internacional em novos contextos locais, e avaliar a probabilidade efetiva de implementação, considerando-se a distância cultural adicionada. Bem como, é importante estar ciente de que, com o aumento da diversidade cultural, o ritmo e o alcance da expansão internacional da multinacional podem ser reduzidos (HUTZSCHENREUTER; VOLL; VERBEKE, 2011).

A visão baseada em recursos pode ser analisada pelas características dos recursos da organização, que podem moderar o efeito da distância sobre o desempenho exportador. Neste estudo, os recursos da organização foram analisados em termos de características da: (1) da equipe de exportação, (2) estrutura organizacional e (3) departamento de 
exportação. Desta forma, apresentamos a Hipótese 2 deste estudo:

- Hipótese 2: Os recursos da organização tem um efeito moderador positivo na relação entre a distância e o desempenho exportador.

- Hipótese 2a: $\mathrm{A}$ equipe de exportação tem um efeito moderador positivo na relação entre a distância e o desempenho exportador.

- Hipótese 2b: A estrutura organizacional tem um efeito moderador positivo na relação entre a distância e o desempenho exportador.

- Hipótese 2c: 0 departamento de exportação tem um efeito moderador positivo na relação entre a distância e o desempenho exportador.

A Figura 1 descreve o modelo referente as hipóteses das relações entre os efeitos das distâncias psíquica, cultural e institucional sobre os determinantes de desempenho exportador, bem como, as influências moderadoras dos recursos da organização: sendo, características da equipe de exportação, estrutura da empresa e o departamento de exportação.

\section{Metodologia}

Este estudo trata de pesquisa descritiva que apresenta determinada situação por meio de medições de um fenômeno, atividade ou evento (HAIR Jr. et al., 2005). As hipóteses foram testadas pela análise de regressão linear múltipla, que "é um modelo mais realista porque, no mundo em que vivemos, a previsão quase sempre depende de vários fatores, não de um só" (HAIR Jr. et al., 2005, p. 326). A análise de variância foi verificada pela ANOVA que "é usada para examinar diferenças estatísticas entre as medidas de dois ou mais grupos" (HAIR Jr. et al., 2005 , p. 321). Foi realizada rotação Varimax para confirmação das dimensões das variáveis independentes de distâncias, sendo, psíquica, institucional e cultural.

Conforme Vieira e Faia (2014), para moderação dos recursos organizacionais sobre a relação entre distância e desempenho de exportação, aplicaram-se os seguintes passos: (1) todas as variáveis foram padronizadas, ou seja, normalizadas com a centralização das variáveis contínuas; (2) criaram-se termos multiplicativos entre as diversas variáveis moderadoras e as variáveis e; (3) testaram-se as regressões destas novas variáveis em conjunto com as moderadoras e independentes.

Foi aplicado método de inserção das variáveis por etapas (stepwise) para cálculo dos efeitos das variáveis moderadoras de recursos da organização sobre a relação das variáveis independentes de distâncias e variáveis dependentes de desempenho de exportação. Não foram realizadas as rotações Varimax, pois tratam-se de variáveis moderadoras.

\subsection{Questionário}

O questionário com 46 perguntas sobre os determinantes de desempenho exportador utilizou escala Likert de 7 pontos, e baseou-se no formulário de Carneiro, Rocha e Silva (2011). A partir da revisão de literatura, as adaptações do questionário tratam dos atributos relacionados às hipóteses de pesquisa, sendo, distâncias, recursos organizacionais e desempenho exportador. O questionário consolidouse após aplicação de pré-teste com gerente de exportação de empresa de grande porte, com objetivo de avaliar a linguagem e a compreensão das questões do instrumento de pesquisa.

O questionário também contou com 12 perguntas sobre produto, país de exportação, viagem ao país, tipo de exportação, perspectivas futuras da atividade, receita total, percentual de exportação e outras, bem como, 8 questões referente a informações gerais da empresa. Os resultados são apresentados no perfil da amostra por meio de estatística descritiva.

\subsection{Constructos e variáveis}

\subsubsection{Variáveis dependentes}

O desempenho exportador foi mensurado a partir da percepção de satisfação dos gestores em relação aos resultados da exportação do principal produto informado pelas empresas, no tocante ao valor total das exportações, margem de lucro, crescimento no volume e, resultados gerais (lucro, volume, posição competitiva, relacionamento com clientes, etc.) de 2011 a 2013.

\section{Tabela 1.1}

Variáveis Dependentes - Desempenho Exportador

\begin{tabular}{c|c|c}
\hline $\begin{array}{c}\text { Desempenho de } \\
\text { Exportação }\end{array}$ & $\begin{array}{c}\text { Exportação para } \\
\text { Outros Países }\end{array}$ & $\begin{array}{c}\text { Expectativa } \\
\text { Resultados Futuro: }\end{array}$ \\
\hline Valor total & Valor total & Resultados gerais \\
\hline Margem de lucro & Crescimento & Quantidade \\
\hline Crescimento & Lucratividade & Crescimento \\
\hline Resultados gerais & $\begin{array}{c}\text { Quantidade X } \\
\text { Concorrente }\end{array}$ & Lucratividade \\
\hline Alfa de Cronbach: 0,81 & 0,69 & 0,88 \\
\hline
\end{tabular}

Fonte: Dados da pesquisa.

Considerando o mesmo período, analisaram-se as percepções referentes aos resultados de exportação 
Tabela 1.2

Variáveis Independentes - Efeitos da Distância

\begin{tabular}{|c|c|c|c|}
\hline Constructo & Variável & Autores & Alfa de Cronbach \\
\hline Distância Psíquica & $\begin{array}{c}\text { Confiança nas pessoas } \\
\text { Grau de profissionalização } \\
\text { Grau de exigência dos produtos }\end{array}$ & Dow and Karunaratna (2006) & 0.66 \\
\hline Distância Cultural & $\begin{array}{l}\text { Costumes e tradições } \\
\text { Ética e códigos de conduta } \\
\text { Normas e práticas de negócios } \\
\text { Comunicação e linguagem }\end{array}$ & Hutzschenreuter et al. (2011) & 0.79 \\
\hline Distância Institucional & $\begin{array}{c}\text { Estrutura política } \\
\text { Estrutura econômica } \\
\text { Estrutura tecnológica } \\
\text { Ambiente regulatório }\end{array}$ & North (1991) & 0.84 \\
\hline
\end{tabular}

Fonte: Dados da pesquisa.

comparativamente as exportações deste produto para outros países em relação ao valor total, crescimento do volume e lucratividade. A quantidade do produto exportado foi analisada em relação aos resultados dos concorrentes. E, para os próximos 3 anos, verificou-se as expectativas de resultados futuros de exportação, considerando uma taxa de câmbio em torno de $\mathrm{R} \$$ 2,10/dólar, referente a resultados gerais, quantidade, crescimento da quantidade e lucratividade. A Tabela 1.1 resume as variáveis dependentes referente ao desempenho exportador, avaliadas nesta pesquisa.

\subsubsection{Variáveis independentes}

As distâncias foram mensuradas a partir dos resultados referentes a costumes locais e tradições do país de exportação, ética e códigos de conduta, normas e práticas de negócios daquele país, comunicação e linguagem do país de exportação, confiança nas pessoas daquele país, grau de profissionalização das pessoas naquele país, estrutura política do país de exportação, estrutura econômica, estrutura tecnológica, ambiente regulatório com a constituição e leis daquele país, e grau de exigência dos produtos daquele país.

A Tabela 1.2 apresenta os constructos das distâncias, variáveis, autores e consistência interna por meio do Alfa de Cronbach. E, a Tabela 1.3 confirma os componentes por meio da rotação Varimax das distâncias.

\subsubsection{Variáveis moderadoras}

Acredita-se que a equipe de exportação é um dos recursos que as empresas podem utilizar para diminuir as distâncias psíquica, cultural e institucional. As equipes foram avaliadas referentes à suas práticas em relação à experiência internacional, habilidade em idiomas, tempo gasto em viagens

Tabela 1.3

Rotação Varimax das Distâncias

\begin{tabular}{l|c|c|c}
\hline \multirow{2}{*}{ Variáveis } & \multicolumn{2}{|c}{ Componentes } \\
\cline { 2 - 4 } & Institucional & Cultural & \\
\hline Estrutura política & 0.89 & & \\
Estrutura econômica & 0.87 & \\
Ambiente regulatório & 0.81 & & \\
Estrutura tecnológica & 0.59 & 0.86 & \\
Ética códigos de conduta & & 0.85 & 0.9 \\
Costumes e tradições & & 0.61 & 0.76 \\
Normas práticas negócios & & & 20.45 \\
Grau de exigência & 31.46 & & 0.66 \\
Confiança nas pessoas & 0.84 & 22.36 & \\
\hline \% Variação & & 0.79 & \\
\hline Alfa Cronbach & & & \\
\hline
\end{tabular}

Fonte: Dados da pesquisa. 
Tabela 1.4

Variáveis Moderadoras - Recursos da Organização

\begin{tabular}{|c|c|c|}
\hline Equipe de Exportação & Estrutura da Organização & Departamento de Exportação \\
\hline Experiência internacional da equipe & Tipo de produto & Grau de autonomia \\
\hline Habilidade em idiomas & Tamanho da empresa & Regras de comportamento \\
\hline Tempo gasto atualmente em viagens internacionais & Idade da empresa & Níveis hierárquicos \\
\hline Conhecimento do mercado do país de exportação & Experiência em exportação & Especialização dos cargos \\
\hline Domínio das formas de negociação & Inovação atual em produtos & \multirow{2}{*}{ Local do departamento } \\
\hline Domínio das finanças internacionais & Competências e capacidades & \\
\hline Domínio dos aspectos econômicos & \multirow{2}{*}{$\begin{array}{l}\text { Planejamento estratégico de } \\
\text { exportação }\end{array}$} & \multirow{2}{*}{ Suporte ao cliente estrangeiro } \\
\hline Domínio da legislação internacional & & \\
\hline Cronbach Alpha: 0.86 & 0.79 & 0.89 \\
\hline
\end{tabular}

Fonte: Dados da pesquisa.

internacionais e conhecimento do mercado do país de exportação. As equipes também foram analisadas referentes ao domínio das formas de negociação, finanças internacionais, aspectos econômicos e legislação internacional do país de exportação.

A estrutura da empresa é outro recurso importante na avaliação das distâncias, sendo mensurada quanto ao tipo de produto, tamanho e idade da empresa, tempo de experiência em exportação, inovação de produtos, competências e capacidades da empresa e o planejamento estratégico de exportação. Outro recurso considerado é o departamento de exportação, relativo ao grau de autonomia, regras de comportamento da equipe, níveis hierárquicos, especialização dos cargos, local do departamento de exportação e o suporte prestado ao cliente estrangeiro. A Tabela 1.4 resume as variáveis moderadoras desta pesquisa.

\subsection{Amostra}

No estado de Santa Catarina constam 1.512 empresas exportadoras ativas em 2013 (MDIC-
Ministério do Desenvolvimento, Indústria e Comércio Exterior, 2014). No mês de junho de 2014, foram enviados 870 questionários pelos correios, e realizados 4 envios para listagem com 636 e-mails e efetuadas 329 ligações telefônicas para solicitar o preenchimento dos formulários desta pesquisa. Obtivemos 49 respostas, sendo que a amostra é composta por $3,24 \%$ empresas produtoras que exportaram seus produtos nos últimos anos.

Em média, as empresas respondentes exportam há 16 anos (de 2 a 60 anos de atividade exportadora) para uma média de 11 países diferentes (até 72 países). No total, as 49 empresas possuem 15.142 funcionários no Brasil e 4.457 empregados no exterior. Os principais produtos exportados pelas empresas respondentes são máquinas e equipamentos mecânicos, peças fundidas em aço, compensados, móveis, portas de madeiras, esquadrias, molduras, têxteis, revestimentos cerâmicos, papel seda, calçados, sucos, duchas eletrônicas e outros.

Para 27 empresas o tipo de exportação, apresentado na Tabela 2, é exportação direta sem agentes. Outras 16 empresas exportam de forma

Tabela 2

Tipo de exportação

\begin{tabular}{|c|c|c|c|c|}
\hline & Freq. & $\%$ & \% válida & $\%$ acum. \\
\hline $\begin{array}{l}\text { Indireta (por meio de trading ou de empresa comercial exportadora ou de agente de } \\
\text { compra sediados no Brasil) }\end{array}$ & 6 & 12.2 & 12.2 & 12.2 \\
\hline $\begin{array}{l}\text { Direta via agentes (por meio de agentes ou representantes da própria empresa } \\
\text { sediados no exterior) }\end{array}$ & 16 & 32.7 & 32.7 & 44.9 \\
\hline $\begin{array}{l}\text { Direta sem agentes (para distribuidores ou para empresas de importação sediados } \\
\text { no exterior; ou para subsidiárias de distribuição ou instalações da sua própria } \\
\text { empresa no exterior; ou diretamente para clientes no exterior) }\end{array}$ & 27 & 55.1 & 55.1 & 100 \\
\hline Total & 49 & 100 & 100 & \\
\hline
\end{tabular}

Fonte: Dados da pesquisa 
Tabela 3

Efeitos das distâncias sobre os desempenhos

\begin{tabular}{rrrr|ccc|ccc}
\hline & \multicolumn{3}{c|}{ Modelo 1a } & \multicolumn{3}{c|}{ Modelo 1b } & \multicolumn{3}{c}{ Modelo 1c } \\
& \multicolumn{1}{c}{ Desempenho Exportação } & \multicolumn{2}{c}{ Exportação Outros Países } & \multicolumn{2}{c}{ Desempenho Futuro } \\
\hline & B & Sig. & VIF & B & Sig. & VIF & B & Sig. & VIF \\
\hline Dist. Psíquica & 0,224 & 0,177 & 1,304 & 0,297 & $\mathbf{0 , 0 6 8}$ & 1,310 & 0,058 & 0,733 & 1,310 \\
Dist. Cultural & 0,108 & 0,549 & 1,587 & 0,096 & 0,585 & 1,593 & 0,051 & 0,789 & 1,593 \\
Dist. Institucional & 0,022 & 0,891 & 1,268 & 0,038 & 0,807 & 1,274 & $-0,040$ & 0,814 & 1,274 \\
\hline $\mathbf{F}$ & & 1,470 & & & 2,336 & & & 0,114 \\
$\mathbf{R}^{2}$ & & 0,091 & & & & 0,135 & & & 0,008 \\
ajustado & & 0,029 & & & 0,077 & & & $-0,059$ \\
\hline
\end{tabular}

Fonte: Dados da pesquisa.

direta via agentes (por meio de agentes ou representantes da própria empresa sediados no exterior). Demais 6 exportam de forma indireta (por meio de trading ou de empresa comercial exportadora, ou de agente de compra sediados no Brasil). Nenhuma utiliza consórcio de exportação (por meio de ação cooperada entre um grupo de empresas).

\section{Estimação do modelo e discussão dos resultados}

A estimação do modelo de análise dos efeitos das distâncias sobre o desempenho exportador dá-se em quatro principais etapas. Na primeira etapa, estimam-se apenas os efeitos das três distâncias
(Tabela 3). Na etapa 2, verifica-se o efeito moderador dos recursos organizacionais (Tabela 4.1). Na etapa 3, procede-se ao teste do efeito moderador da equipe de exportação (Tabela 4.2). Nas etapas 4 e 5, respectivamente testam-se os efeitos da estrutura organizacional (Tabela 4.3) e do departamento de exportação (Tabela 4.4).

As hipóteses $\mathrm{H} 1, \mathrm{H} 1 \mathrm{a}, \mathrm{H} 1 \mathrm{~b}$ e $\mathrm{H} 1 \mathrm{c}$ tratam dos efeitos da distância sobre o desempenho exportador, em que, quanto maiores as distâncias psíquica, cultural e institucional entre o país de origem da empresa exportadora e o país de importação o qual é o destino dos produtos, menor é o desempenho exportador da organização. E, no instrumento de pesquisa respondido pelas 49 empresas produtoras

Tabela 4.1

Moderação dos recursos organizacionais sobre a relação entre distância e desempenho

\begin{tabular}{|c|c|c|c|c|c|c|}
\hline & \multicolumn{2}{|c|}{$\begin{array}{c}\text { Modelo 1a } \\
\text { Desempenho Exportação }\end{array}$} & \multicolumn{2}{|c|}{$\begin{array}{c}\text { Modelo 1b } \\
\text { Exportação Outros Países }\end{array}$} & \multicolumn{2}{|c|}{$\begin{array}{c}\text { Modelo 1c } \\
\text { Desempenho Futuro }\end{array}$} \\
\hline & B & Sig. & B & Sig. & B & Sig. \\
\hline Distância Psíquica & ns & ns & ns & ns & ns & ns \\
\hline Distância Cultural & ns & ns & ns & ns & ns & ns \\
\hline Distância Institucional & ns & ns & ns & ns & ns & ns \\
\hline Equipe de Exportação & ns & ns & ns & ns & 0.293 & $0.041^{*}$ \\
\hline Estrutura Organização & 0.429 & $0.002^{*}$ & ns & ns & ns & ns \\
\hline Departamento Export. & ns & ns & ns & ns & ns & ns \\
\hline Psíquica X Equipe & ns & ns & 0.410 & $0.003^{*}$ & ns & ns \\
\hline Cultural X Equipe & ns & ns & ns & ns & ns & ns \\
\hline Institucional X Equipe & ns & ns & ns & ns & ns & ns \\
\hline Psíquica X Estrutura & ns & ns & ns & ns & ns & ns \\
\hline Cultural X Estrutura & ns & ns & ns & ns & ns & ns \\
\hline Institucional X Estrutura & ns & ns & ns & ns & ns & ns \\
\hline Psíquica X Depto & ns & ns & ns & ns & ns & ns \\
\hline Cultural X Depto & ns & ns & ns & ns & ns & ns \\
\hline Institucional X Depto & ns & ns & ns & ns & ns & ns \\
\hline $\mathrm{F}$ & & 10.600 & & 9.514 & & 4.423 \\
\hline $\mathrm{R}^{2}$ & & 0.187 & & 0.168 & & 0.086 \\
\hline ajustado & & 0.170 & & 0.151 & & 0.067 \\
\hline
\end{tabular}

Método de inserção das variáveis: Em etapas (Critérios: Probabilidade de $\mathrm{F}$ a ser inserido $<=, 090$, Probabilidade de $\mathrm{F}$ a ser removido $>=, 100) .{ }^{* *}$ para $p<0,01$; ${ }^{*}$ para $p<0,05$; ns: não significativo

Fonte: Dados da pesquisa. 
Tabela 4.2

Moderação da equipe de exportação sobre a relação entre distância e desempenho

\begin{tabular}{|c|c|c|c|c|c|c|}
\hline & \multicolumn{2}{|c|}{$\begin{array}{c}\text { Modelo 1a } \\
\text { Desempenho Exportação }\end{array}$} & \multicolumn{2}{|c|}{$\begin{array}{c}\text { Modelo 1b } \\
\text { Exportação Outros Países }\end{array}$} & \multicolumn{2}{|c|}{$\begin{array}{c}\text { Modelo 1c } \\
\text { Desempenho Futuro }\end{array}$} \\
\hline & B & Sig. & B & Sig. & B & Sig. \\
\hline Distância Psíquica & ns & ns & ns & ns & ns & ns \\
\hline Distância Cultural & ns & ns & ns & ns & ns & ns \\
\hline Distância Institucional & ns & ns & ns & ns & ns & ns \\
\hline Equipe de Exportação & ns & ns & ns & ns & 0.293 & $0.041 *$ \\
\hline Psíquica X Equipe & 0.322 & $0.024^{*}$ & 0.410 & $0.003^{*}$ & ns & ns \\
\hline Cultural X Equipe & ns & ns & ns & ns & ns & ns \\
\hline Institucional X Equipe & ns & ns & ns & ns & ns & ns \\
\hline$F$ & & 5.435 & & 9.514 & & 4.423 \\
\hline $\mathrm{R}^{2}$ & & 0.106 & & 0.168 & & 0.086 \\
\hline ajustado & & 0.086 & & 0.151 & & 0.067 \\
\hline
\end{tabular}

Método de inserção das variáveis: Em etapas (Critérios: Probabilidade de $\mathrm{F}$ a ser inserido $<=, 090$, Probabilidade de $\mathrm{F}$ a ser removido $>=, 100)$.** para $p<0,01$; ${ }^{*}$ para $p<0,05$; ns: não significativo.

Fonte: Dados da pesquisa.

exportadoras, as questões referente as distâncias foram mensuradas com escalas referente ao desempenho de exportação, sendo de 1 (forte barreira) até 7 (forte incentivo).

Conforme Tabela 3, somente a distância psíquica (H1a) se relaciona com o desempenho de exportação do mesmo produto para outros países (Sig. 0,068). E, constata-se que nenhuma das demais médias das variáveis dos constructos das distâncias psíquica, cultural e institucional apresentaram significância estatística com as médias das variáveis de determinantes de desempenho, sendo, desempenho entre 2011 e 2013, desempenho exportador comparativamente com outros países, e expectativa de desempenho exportador para os próximos 3 anos.
Analisando o conjunto de variáveis independentes, o F também possui pouca significância (Sig. 1,470, 2,336 e 0,114), tampouco, a variação nas variáveis independentes ( $R^{2}$ 0,091, 0,135 e 0,008), explicando, no máximo, apenas $13,5 \%$ do desempenho exportador comparativamente com outros países. Portanto, os resultados não confirmam as hipóteses $\mathrm{H} 1 \mathrm{~b}$ e H1c, para a amostra desta pesquisa.

A Tabela 4.1 apresenta a análise da influência dos recursos organizacionais sobre a relação entre distância e desempenho. Foi utilizada a regressão linear múltipla com método de inserção das variáveis em etapas (stepwise) e, na amostra pesquisada, verificou-se relação entre a estrutura da organização e o desempenho de exportação (Sig. 0,002). A variável multiplicadora "distância psíquica $X$ equipe de exportação" também apresenta relação com a

Tabela 4.3

Moderação da estrutura da organização sobre a relação entre distância e desempenho

\begin{tabular}{|c|c|c|c|c|c|c|}
\hline & \multicolumn{2}{|c|}{$\begin{array}{c}\text { Modelo 1a } \\
\text { Desempenho Exportação }\end{array}$} & \multicolumn{2}{|c|}{$\begin{array}{c}\text { Modelo 1b } \\
\text { Exportação Outros Países }\end{array}$} & \multicolumn{2}{|c|}{$\begin{array}{c}\text { Modelo 1c } \\
\text { Desempenho Futuro }\end{array}$} \\
\hline & B & Sig. & B & Sig. & $\mathrm{B}$ & Sig. \\
\hline Distância Psíquica & ns & ns & 0.352 & $0.013^{*}$ & ns & ns \\
\hline Distância Cultural & ns & ns & ns & ns & ns & ns \\
\hline Distância Institucional & ns & ns & ns & ns & ns & ns \\
\hline Estrutura Organização & 0.429 & $0.002^{*}$ & ns & ns & ns & ns \\
\hline Psíquica X Estrutura & ns & ns & ns & ns & ns & ns \\
\hline Cultural X Estrutura & ns & ns & ns & ns & ns & ns \\
\hline Institucional X Estrutura & ns & ns & ns & ns & ns & ns \\
\hline $\mathrm{F}$ & & 10.600 & & 6.628 & & ns \\
\hline $\mathrm{R}^{2}$ & & 0.187 & & 0.124 & & ns \\
\hline ajustado & & 0.170 & & 0.105 & & ns \\
\hline
\end{tabular}

Método de inserção das variáveis: Em etapas (Critérios: Probabilidade de $\mathrm{F}$ a ser inserido $<=, 090$, Probabilidade de $\mathrm{F}$ a ser removido $>=, 100)$.* para $p<0,01 ;{ }^{*}$ para $p<0,05$; ns: não significativo

Fonte: Dados da pesquisa. 
exportação para países que não são o destino principal das exportações (Sig. 0,003). Por fim, a equipe de exportação apresenta relação com a expectativa de desempenho futuro nos próximos três anos (Sig. 0,041).

Para análise da influência da equipe de exportação sobre a relação entre a distância e o desempenho de exportação, a Tabela 4.2 apresenta este efeito moderador. A variável multiplicadora "distância psíquica X equipe de exportação" exibe relaçãoempenho de exportação (Sig. 0,024) e a exportação para outros países (Sig. 0,003). E, a equipe de exportação também apresenta relação com a expectativa de desempenho futuro (Sig. 0,041).

A moderação da estrutura da organização destaca relação com o desempenho de exportação (Sig. 0,002), conforme Tabela 4.3, assim como, a distância psíquica aponta relação com a exportações para outros países (Sig. 0,013).

Por fim, na Tabela 4.4, a análise da influência do departamento de exportação sobre a relação entre a distância e o desempenho, apresenta relação entre a variável multiplicadora "distância psíquica $X$ departamento de exportação" e o desempenho exportador (Sig. 0,031) e relação entre a distância psíquica e a exportação para outros países (Sig. 0,013). desempenho exportador atual, exportação para outros países e expectativa de resultados futuros também apresentaram bons indicadores de Alfa de Cronbach. Finalmente, os constructos das variáveis moderadoras de equipe de exportação, estrutura da organização e departamento de exportação, igualmente possuem validade interna, o que possibilita a análise das hipóteses deste estudo.

Somente a distância psíquica possui relação positiva com o desempenho de exportação para outros países (Sig. 0,068). Portanto, para a amostra pesquisada, apresentaram-se apenas evidências sobre a hipótese $\mathrm{H} 1 \mathrm{a}$. As demais hipóteses $\mathrm{H} 1, \mathrm{H} 1 \mathrm{~b}$ e H1c não foram demonstradas, pois, não há significância entre as distâncias cultural e institucional em relação aos desempenhos de exportação atual, para outros países e desempenho futuro.

As hipóteses $\mathrm{H} 2, \mathrm{H} 2 \mathrm{a}, \mathrm{H} 2 \mathrm{~b}$, e H2c tratam do efeito moderador dos recursos da organização sobre a relação entre a distância e o desempenho exportador. Na análise de todas as variáveis moderadoras em conjunto, a estrutura da organização assinala relação positiva com o desempenho de exportação (Sig. 0,002), confirmando parcialmente a hipótese H2a. Assim como, a equipe de exportação destaca relação

\section{Tabela 4.4}

Moderação do departamento de exportação sobre a relação entre distância e desempenho

\begin{tabular}{|c|c|c|c|c|c|c|}
\hline & \multicolumn{2}{|c|}{$\begin{array}{c}\text { Modelo 1a } \\
\text { Desempenho Exportação }\end{array}$} & \multicolumn{2}{|c|}{$\begin{array}{c}\text { Modelo 1b } \\
\text { Exportação Outros Países }\end{array}$} & \multicolumn{2}{|c|}{$\begin{array}{c}\text { Modelo 1c } \\
\text { Desempenho Futuro }\end{array}$} \\
\hline & B & Sig. & B & Sig. & B & Sig. \\
\hline Distância Psíquica & ns & ns & 0.352 & $0.013^{*}$ & ns & ns \\
\hline Distância Cultural & ns & ns & ns & ns & ns & ns \\
\hline Distância Institucional & ns & ns & ns & ns & ns & ns \\
\hline Departamento Export. & ns & ns & ns & ns & ns & ns \\
\hline Psíquica X Depto & 0.309 & $0.031^{*}$ & ns & ns & ns & ns \\
\hline Cultural X Depto & ns & ns & ns & ns & ns & ns \\
\hline Institucional X Depto & ns & ns & ns & ns & ns & ns \\
\hline $\mathrm{F}$ & & 4.968 & & 6.628 & & ns \\
\hline $\mathrm{R}^{2}$ & & 0.097 & & 0.124 & & ns \\
\hline ajustado & & 0.078 & & 0.105 & & ns \\
\hline
\end{tabular}

Método de inserção das variáveis: Em etapas (Critérios: Probabilidade de $\mathrm{F}$ a ser inserido $<=, 090$, Probabilidade de $\mathrm{F}$ a ser removido $>=, 100)$.** para $p<0,01$; * para $p<0,05$; ns: não significativo

Fonte: Dados da pesquisa.

\section{Considerações Finais}

$\mathrm{Na}$ amostra pesquisada, pode-se afirmar que os constructos dos efeitos das distâncias psíquica, cultural e institucional possuem consistência interna. Da mesma forma, as variáveis dependentes de positiva com a expectativa de desempenho futuro (Sig. 0,041), admitindo em parte a hipótese $\mathrm{H} 2 \mathrm{c}$. Referente a hipótese $\mathbf{H} \mathbf{2 b}$, apenas a variável multiplicadora demonstrou relação significativa.

$\mathrm{Na}$ análise somente do efeito moderador da equipe 
de exportação sobre a relação entre distância e desempenho, demonstram-se evidências sobre a hipótese H2c, em que a equipe de exportação possui relação positiva com o desempenho futuro (Sig. 0,041 ). A variável multiplicadora "distância psíquica $X$ equipe" apontou significância com o desempenho de exportação e, também, com a exportação para outros países, confirmando em parte o efeito moderador previsto nas hipóteses $\mathrm{H} 2 \mathrm{a}$ e $\mathrm{H} 2 \mathrm{~b}$.

No exame da influência moderadora da estrutura da organização sobre a relação entre distância e desempenho, considera-se parcialmente aceita a hipótese H2a, pois, a estrutura da organização aponta relação positiva com o desempenho exportador (Sig. 0,002). Nesta moderação, a distância psíquica apresenta relação positiva com a exportação para outros países (Sig. 0,013).

E, por fim, a moderação do departamento de exportação sobre a relação entre distância e desempenho não apresenta resultados que coadunam com as hipóteses do estudo. Apenas a variável multiplicadora "distância psíquica $X$ departamento" possui relação com o desempenho exportador e, a distância psíquica apresenta significância com a exportação para outros países.

O presente estudo não corrobora as conclusões de He, Brouthers e Filatotchev (2012), em que o desempenho superior das exportações está alinhado com os fatores baseados em recursos e a distância institucional. A análise empírica sugere que, depois de considerar a influência moderadora da distância institucional, alinhando os recursos internos com o canal de exportação, há maior desempenho das exportações (HE; BROUTHERS; FILATOTCHEV, 2012).

Ainda, esta pesquisa não confirma os achados de Carneiro, Rocha e Silva (2011) com 389 grandes exportadores brasileiros de produtos manufaturados, que verificou efeito negativo moderado da distância psíquica na rentabilidade das exportações. Segundo estes autores, as receitas de exportação mostraram uma forte associação positiva com o estado da atividade exportadora e uma forte associação negativa com barreiras no país de acolhimento. A rentabilidade das exportações mostrou uma forte associação positiva com a sistematização de planejamento de exportação e uma associação negativa moderada com a distância psíquica e com barreiras no país de acolhimento (CARNEIRO; ROCHA; SILVA, 2011).
Por fim, não é possível comparar o estudo de Hutzschenreuter, Voll e Verbeke (2011), pois este trata dos efeitos da distância cultural existente e adicionada, em relação a diversificação e a expansão internacional no período subsequente.

As conclusões elencadas limitam-se a amostra pesquisada, e conforme apresentado, precisam-se considerar as quantidades das variações nas variáveis dependentes associadas com todas as variáveis independentes consideradas em conjunto (medida de adequação). A principal limitação deste estudo foi o baixo interesse das empresas exportadoras em participar da pesquisa e responder o questionário enviado pelos correios e listagem de e-mails.

\section{Referências}

- AUlAKH, P.; KOTABE, M.; TEEGEN, H. Export Strategies and Performance of Firms from Emerging Economies: Evidence from Brazil, Chile, and Mexico. Academy of Management Journal, v.43, n.3, p.342-361, 2000.

- BARKEMA, Harry; BELL, John; PENNINGS, J. M. E. Foreign entry, cultural barriers and learning. Strategic Management Journal, v. 17, n. 2, p. 151-166, 1996.

- BARNEY, Jay; WRIGHT, Mike; KETCHEN, David J. The resource-based view of the firm: Ten years after 1991. Journal of Management, v. 27, n. 6, p. 625-641, $2001 . \quad$ DOI $10.1177 / 014920630102700601$

- BARNEY, Jay. Firm resources and sustained competitive advantage. Journal of Management, $v$. 17, n. 1, p. 99-120, 1991.

- BECKERMAN, W. Distance and the pattern of intraEuropean trade. The Review of Economics and Statistics, v. 38, n. 1, p. 31-40, 1956. DOI $10.2307 / 1925556$

- CARNEIRO, Jorge; ROCHA, Angela da; SILVA, Jorge Ferreira da. Determinants of export performance: a study of large Brazilian manufacturing firms. BAR - Brazilian Administration Review, v. 8, n. 2, p. 107132, 2011. DOI 10.1590/s180776922011000200002

- DICHTL, Erwin; KOEGLMAYR, Hans-Georg; MUELLER, Stefan. International orientation as a precondition for export success. Journal of International Business Studies, v. 21, n. 1, p. 23-40, 1990.

- DIMAgGIO, P. J.; POWELL, W. W. The iron cage revisited: Institutional isomorphism and collective 
rationality in organizational fields. American Sociological Review, v. 48, n. 2, p. 147-160, 1983. DOI 10.2307/2095101

- DOW, Douglas; KARUNARATNA, Amal. Developing a multidimensional instrument to measure psychic distance stimuli. Journal of International Business Studies, v. 37, n. 5, p. 578-602, 2006.

- GHEMAWAT, Pankaj. Distance still matters. Harvard Business Review, v. 79, n. 8, p. 137-147, 2001.

- haIR JR, J. F.; BABIN, B.; MONEY, A. H.; SAMUEL, P. Fundamentos de métodos de pesquisa em administração. Porto Alegre: Bookman, 2005.

- HÅKANSON, Lars; AMBOS, Björn. The antecedents of psychic distance. Journal of International Management, v. 16, n. 3, p. 195-210, 2010. DOI 10.1016/j.intman.2010.06.001

- HE, Xinming; BROUTHERS, Keith D.; FILATOTCHEV, Igor. Resource-based and institutional perspectives on export channel selection and export performance. Journal of Management, $v$. 39 , n. 1, p. 27-47, 2012. DOI 10.1177/0149206312445926

- HENRIQUeS, Irene; SADORSKY, Perry. The determinants of an environmentally responsive firm: an empirical approach. Journal of Environmental Economics and Management, v. 30, n. 3, p. 381-395, 1996

- HOFFMAN, Andrew J. Linking organizational and field-level analyses the diffusion of corporate environmental practice. Organization \& Environment, v. 14, n. 2, p. 133-156, 2001.

- HUTZSChENREUTER, Thomas; VOLL, Johannes C.; VERBEKE, Alain. The impact of added cultural distance and cultural diversity on international expansion patterns: A Penrosean perspective. Journal of Management Studies, v. 48, n. 2, p. 305 329, 2011. DOI 10.1111/j.14676486.2010.00966.x

- JOHANSON, Jan; VAHLNE, Jan-Erik. The internationalization process of the firm - a model of knowledge development and increasing foreign market commitments. Journal of International Business Studies, v. 8, n. 1, p. 23-32, 1977. DOI 10.1057/palgrave.jibs.8490676

- JOHANSON, Jan; VAHLNE, Jan-Erik. The Uppsala internationalization process model revisited: From liability of foreignness to liability of outsidership. Journal of International Business Studies, v. 40, n. 9 , p. 1411-1431, 2009. DOI 10.1057/jibs.2009.24
- KIRKMAN, Bradley L.; LOWE, Kevin B.; GIBSON, Cristina B. A quarter century of culture's consequences: A review of empirical research incorporating Hofstede's cultural values framework. Journal of International Business Studies, v. 37, n. 3, p. 285-320, 2006.

- LeONIDOU, L. C. et al. Marketing strategy determinants of export performance: a metaanalysis. Journal Business Research, vol. 55, n.1, p.51-67, 2002.

- LEUnG, K.; BHAGAT, R. S.; BUCHAN, N. R., EREZ, M.; GIBSON, C. B. Culture and international business: recent advances and their implications for future research. Journal of International Business Studies, v. 36, n.4, p. 357-378, 2005. DOI 10.1057/palgrave.jibs. 8400150

- MADSEN, T. K. Successful export marketing management: some empirical evidence. International Marketing Review, vol. 6, n. 4, p. 4157, 1989.

- MEYER, J. W.; ROWAN, B. Institutionalized organizations: Formal structure as myth and ceremony. American Journal of Sociology, v. 83, n. 2, p. 340, 1977. DOI 10.1086/226550

- MEYER, Klaus E.; MUDAMBI, Ram; NARULA, Rajneesh. Multinational enterprises and local contexts: the opportunities and challenges of multiple embeddedness. Journal of Management Studies, v. 48, n. 2, p. 235-252, 2011. DOI 10.1111/j.1467-6486.2010.00968.x

- MINISTÉRIO DO DESENVOLVIMENTO, INDÚSTRIA E COMÉRCIO EXTERIOR. Empresas brasileiras exportadoras por países de destino ou por Unidade da Federação. 2014. Disponível em: http://www.mdic.gov.br/sitio/interna/interna.php ?area $=5 \&$ menu $=1444 \&$ refr $=603$. Acesso em: 13 jan. 2014.

- NORTH, D. C. Institutions. The Journal of Economic Perspectives, v. 5, n. 1, p. 97-112, 1991. DOI 10.1257/jep.5.1.97

- OLIVER, C. Sustainable competitive advantage: Combining institutional and resource-based views. Strategic Management Journal, v. 18, n. 9, p. 697713, 1997. DOI 10.1002/(sici)10970266(199710)18:9<697::aid-smj909>3.0.co;2-c

- $\quad$ PENG, Mike W.; WANG, Denis YL; JIANG, Yi. An institution-based view of international business strategy: A focus on emerging economies. Journal of International Business Studies, v. 39, n. 5, p. 920936, 2008.

- PlATCHEK, Renata Bertoldi; FLORIANI, Dinorá Eliete; BORINI, Felipe Mendes. A Influência do 
Grau de Internacionalização no Desempenho das Empresas Têxteis. Revista Gestão Organizacional, v. 5, n. 1, p. 70-81, 2012

- SHENKAR, Oded. Cultural distance revisited: Towards a more rigorous conceptualization and measurement of cultural differences. Journal of International Business Studies, v. 32, n. 3, p. 519535, 2001. DOI 10.1057/palgrave.jibs.8490982
- SOUSA, Carlos MP; BRADLEY, Frank. Cultural distance and psychic distance: two peas in a pod? Journal of International Marketing, v. 14, n. 1, p. 4970, 2006.

- VIEIRA, Valter Afonso; FAIA, Valter da Silva. Efeitos Moderadores Duplos e Triplos na Análise de Regressão. XXXVIII Encontro da Anpad. Rio de Janeiro, 2014.

\title{
Sobre os autores
}

- Givanildo Silva é Doutorando em Administração pela Fundação Universidade Regional de Blumenau-FURB, Blumenau, SC,Brasil.E-mail: givanildo@givanildo.adm.br

- Mohamed Amal é Doutor em Engenharia da Produção. Professor no Programa de Pós-graduação em Administração e no departamento de Economia na Fundação Universidade Regional de Blumenau - FURB, Blumenau, SC, Brasil.E-mail: mohamedamal.amal@gmail.com

- Gérson Tontini é Doutor em Engenharia Mecânica. Professor titular da Fundação Universidade Regional de Blumenau -FURB, Blumenau, SC, Brasil.E-mail: gersontontini@gmail.com

- Gustavo da Rosa Borges é Doutor em Administração. Professor titular da Universidade Federal do Pampa-UFP,Dom Pedrito,RS,Brasil.E-mail: gustavodarosaborges@gmail.com

\section{Effects of distances and company resources for enterprise export performance}

\author{
Givanildo Silva ${ }^{A}$, Mohamed Amal ${ }^{A}$, Gérson Tontini ${ }^{A}$ and Gustavo da Rosa Borges ${ }^{B}$ \\ ${ }^{A}$ Fundação Universidade Regional de Blumenau - FURB, Blumenau, SC, Brazil \\ ${ }^{B}$ Universidade Federal do Pampa - UFP, Dom Pedrito, RS, Brazil
}

\section{ARTICLE DETAILS}

Article history:

Received 14 January de 2015

Accepted 27 July 2015

Available online in 19 December 2015

Double Blind Review System

\section{Scientific Editor}

Felipe Mendes Borini

\section{Keywords:}

Institutional distance

Export determinants

Company resources

\section{ABSTRACT}

The purpose of this article is to evaluate the effect of distance on the export performance of companies in Santa Catarina, and to what extent this effect is moderated by organizational resource characteristics. Multiple linear regression and variance analysis were used for a perception survey of export managers with a final sample of 49 exporting producers. The constructs showed internal validity and allowed the data to be analyzed. The results only revealed evidence regarding the effect of psychic distance, showing a positive relationship with export performance. Also, the model estimation showed that the organization's resources moderate the relation between distance and export performance. Finally the study also shows that the export team and the organizational structure moderate the effect of distance on the performance and future performance expectations of the companies.

(c) 2015 Internext | ESPM. All rights reserved! 